\title{
ADHD AND MOTOR PERCEPTION DYSFUNCTION COMORBIDITY
}

The epidemiology, co-morbidity and overlap of attention deficit hyperactivity disorder (ADHD) and deficits in attention, motor control and perception (DAMP), as defined in Scandinavia, were assessed in a total population of 589 6-year-old children screened for neurodevelopmental and neuropsychiatric disorders at the Skovde Central Hospital, and the University of Goteborg, Sweden. Among 63 children (10.7\%) with identified disorders, the prevalence rates for ADHD and DAMP were 2.4-4\% and 5.3-6.9\%, respectively. One in four to three in four of children with DAMP had ADHD. Attention problems were more pronounced in the ADHD group, but overactivity and impulsivity did not distinguish ADHD from DAMP. Children with DAMP had, by definition, more deficits in perception and motor function. (Landgren $M$, Pettersson R, Kjellman B, Gillberg C. ADHD, DAMP and other neurodevelopmental/psychiatric disorders in 6-year-old children: epidemiology and co-morbidity. Dev Med Child Neurol Oct 1996;38:891-906). (Respond: Dr Magnus Landgren, Department of Paediatrics, Skovde Central Hospital, S-541 85 Skovde, Sweden).

COMMENT. The Scandinavian syndrome of DAMP emphasizes the association of neurological signs of motor dysfunction, perceptual dysfunction, and attention deficits, whereas the current American DSM criteria for ADHD include only symptoms of inattentiveness, hyperactivity, and impulsivity, excluding reference to tests for neurological and perceptual dysfunction. The overlap of these syndromes and the higher prevalence of DAMP compared to ADHD suggests that the neurological examination and tests for perceptual dysfunction should form an integral part of the criteria for diagnosis of the attention deficit disorders (ADHD). A return to the former minimal brain dysfunction (MBD) criteria, in addition to symptoms of ADHD, would allow a more objective diagnosis and earlier recognition and remediation of associated motor incoordination and perceptual deficits.

Conflicting parent and teacher reports of problem behaviors were noted in children with reading disabilities and/or ADHD in a study at the University of Houston. (Pisecco S et al. I Am Acad Child Adolesc Psychiatry Nov 1996;35:1477-1484). In evaluating a child for ADHD, it is important to obtain both teacher and parent reports.

\section{PRENATAL AND PERINATAL DISORDERS}

\section{INTRAVENTRICULAR HEMORRHAGE AND COGNITION}

The effects of premature birth-related subependymal and mild intraventricular hemorrhage (S/IVH) on specific cognitive abilities in 2year-old children were investigated at the Perinatology Center, New York Hospital, and Cornell and New York University Medical Colleges. Of 82 children included in the study, 27 had premature births complicated by Grade I or II hemorrhages, 28 prematurely born children had normal neonatal ultrasound, and 27 were born at term without complications. The premature group with $\mathrm{S} / \mathrm{IVH}$ at birth performed significantly less well than children without hemorrhage on a measure of memory for location and on ability to change response set. Both groups of prematurely born children performed less well than full term children on systematic search for an object when the order of hiding was reversed. All groups performed equally on a visual attention task 
and on the global Bayley mental ability scores. (Ross G, Boatright S, Auld PAM, Nass R. Specific cognitive abilities in 2-year-old children with subependymal and mild intraventricular hemorrhage. Brain Cogn Oct 1996;32:1-13). (Reprints: Dr Gail Ross, Perinatology Center, New York Hospital, 525 East 68 th Street, New York, NY 10021).

COMMENT. Prior testing of these prematures at age 10 months had shown that S/IVH affected global mental ability and habituation to visual patterns, and prematurity was associated with poorer memory for location. When reevaluated at 2 years, the premature groups with or without hemorrhage did not differ on the visual attention task, but prematures with S/IVH did poorly on the memory for location task and ability to change response set. Memory for location is a function of the caudate nucleus and thalamus and frontal cortex, areas affected by subependymal and intraventricular hemorrhage of prematurity. Fronto-striatal structural changes in the MRI have been demonstrated in patients with ADHD. (Denckla MB. In: Progress in Pediatric Neurology II, Chicago, PNB Publ, 1994:173-176). At a later age, the incidence of ADHD in the S/IVH affected children will be of interest.

Indomethacin prophylaxis against IVH in very low birth weight infants did not result in adverse cognitive or motor outcomes at 36 months, in a study at Yale University School of Medicine. (Ment LR et al. Pediatrics Oct 1996;98:714-718). The authors suggest that the early administration of intravenous low-dose indomethacin to neonates weighing $1250 \mathrm{~g}$ or less is beneficial and does not cause neurodevelopmental delay. However, Dr Henrietta Bada, University of Tennessee, Memphis, advises caution, and slow infusion, because of reported acute cerebral effects. (Commentary. Routine indomethacin prophylaxis: has the time come? Pediatrics Oct 1996;98:784-785).

\section{EARLY DETECTION OF CEREBRAL PALSY}

An Early Motor Pattern Profile (EMPP), consisting of 15 tests of muscle tone, reflexes, and movement, organized in a standardized format, was used to identify children with cerebral palsy (CP) in the first year of life at the University of Illinois at Chicago, College of Medicine at Peoria. The items evaluated included head lag, hip abduction, tonic neck reflex, fisting, scissoring, and toe-walking, and a three-point scoring system was applied to each item. In 1247 high-risk infants from a neonatal intensive care unit followed for 36 months, the EMPP identified children at risk for CP by 6 to 12 months of age. The optimal cutoff score of points at 6 months was 10 , and at 12 months it was 4 . The predictive value was approximately 90\%. (Morgan AM, Aldag JC. Early identification of cerebral palsy using a profile of abnormal motor patterns. Pediatrics Oct 1996;98:692-697). (Reprints: Dr Andrew M Morgan, Department of Pediatrics, St Francis Medical Center, 530 NE Glen Oah Ave, Peoria, IL 61637).

COMMENT. The authors emphasize that the EMPP is not a method of diagnosis, but only a step toward more formal evaluation by a pediatric neurologist. False positive and negative results are always a problem with screening procedures of this type, and their sensitivity is dependent on the skill and expertise of the examiner.

Progressive deterioration of dyskinetic cerebral palsy in adult life is reported in the majority of 20 patients examined between 20 and 40 years of age at the Institute of Neurology, London. (Fletcher NA, Marsden CD. Dyskinetic cerebral palsy: a clinical and genetic study. Dev Med Child Neurol 